\title{
High-density plasma production with potential confinement in the GAMMA 10 tandem mirror*
}

\author{
M. Ichimura, ${ }^{\dagger}$ T. Cho, M. Hirata, H. Hojo, K. Ishii, A. Itakura, I. Katanuma, J. Kohagura, \\ Y. Nakashima, T. Saito, T. Tamano, S. Tanaka, Y. Tatematsu, K. Yatsu, \\ and M. Yoshikawa \\ Plasma Research Center, University of Tsukuba, Tsukuba, Ibaraki 305-8577, Japan
}

(Received 23 October 2000; accepted 19 December 2000)

\begin{abstract}
The improvement of potential confinement was attained in the GAMMA 10 tandem mirror [Phys. Rev. Lett. 55, 939 (1985); Proceedings of the 13th International Conference on Plasma Physics and Controlled Nuclear Fusion Research, Washington, 1990 (International Atomic Energy Agency, Vienna, 1991), Vol. 2, p. 539] by axisymmetrization of heating systems for the plasma production, heating, and potential formation. A significant increase of the density and diamagnetism by the potential confinement was observed. In the previous experiment, it was difficult to increase the central cell density higher than $2.7 \times 10^{18} \mathrm{~m}^{-3}$. One of the possible mechanisms is the density clamping due to the eigenmode formation of the ion-cyclotron-range of frequency (ICRF) waves in the axial direction. With high harmonic ICRF waves (RF3), the experiments to overcome this problem have been performed. In preliminary experiments with RF3 and NBI the maximum density of $4 \times 10^{18} \mathrm{~m}^{-3}$ was attained. (c) 2001 American Institute of Physics. [DOI: 10.1063/1.1350962]
\end{abstract}

\section{INTRODUCTION}

The high density and long pulse operation is the important subject in the tandem mirror experiments. The formation of the confining potential in the high density plasma is also the key issue that should be confirmed on the present experiments.

In the GAMMA 10 tandem mirror, the formation of the plug and thermal barrier potentials have been realized ${ }^{1}$ and the high ion temperature of above $10 \mathrm{keV}$ has been achieved. ${ }^{2}$ However, the density was relatively low on such high performance discharges. Ion cyclotron range of frequency (ICRF) waves are used for the plasma production and heating and electron cyclotron heating $(\mathrm{ECH})$ are used for the potential formation. The magnetohydrodynamic (MHD) stability is maintained by also ICRF-heated plasmas in the minimum- $B$ anchor cells. The emission of D-D fusion neutrons has been observed in the experiment with mixed gases of hydrogen and deuterium ${ }^{3}$ with strong ICRF heating by keeping MHD stability. After improvements of the axisymmetry of the heating patterns and plasma uniformity, the density increase due to the potential confinement has been clearly observed. ${ }^{4}$ The increasing rate depends on the initial plasma density. When the density before the potential formation is low, a large density increase is observed. When the initial density is relatively high, the increasing rate becomes small. It was difficult to obtain the central cell density higher than $2.7 \times 10^{18} \mathrm{~m}^{-3}$ in the GAMMA 10 tandem mirror. ${ }^{5}$ Under the present experimental conditions, there is a saturation value in the attained density. The mechanism is not well understood yet. It was suggested that the density could not increase due to the lack of the gas fueling in the core region

*Paper UI1 6, Bull. Am. Phys. Soc. 45, 290 (2000).

${ }^{\dagger}$ Invited speaker. and/or the density was clamped by the eigenmode formation of the ICRF waves in the axial direction. The density increase due to the mode excitation has been observed in many laboratory plasma sources with helicon discharges. ${ }^{6,7}$ Recently, the peak density of $4 \times 10^{18} \mathrm{~m}^{-3}$ was obtained on the combination experiments with the neutral beam injections (NBI) and ICRF waves that have the frequency of much higher than the ion cyclotron frequency. In those experiments, NBI was effective for the density increase in the anchor region. The ICRF waves with near ten times ion cyclotron frequency were effectively used for the high-density plasma production. The density clamping will become weak due to the excitation of several eigenmodes with different radial mode numbers by using high harmonic ICRF waves. The density increased $35 \%$ of the initial density in the highdensity experiments with the potential formation. ${ }^{8}$

In this manuscript, the experimental setup is shown in Sec. II and eigenmodes formation of the fast Alfvén waves and preliminary results with high-harmonic ICRF waves are described in Sec. III. The high-density operation of the GAMMA 10 is described in Sec. IV. The summary is described in Sec. V.

\section{EXPERIMENTAL SETUP}

\section{A. GAMMA 10 tandem mirror}

The schematic drawing of the coil system of the GAMMA 10 and the magnetic field line are shown in Fig. 1. The locations of the heating systems are also illustrated. The central cell and plug/barrier cells have the axisymmetric mirror configuration and the anchor cells located between central cell and plug/barrier cells have the minimum- $B$ mirror configuration with nonaxisymmetric magnetic fields. The quadrapole type minimum- $B$ configuration has essentially noncircular and extremely elongated fan regions on both 


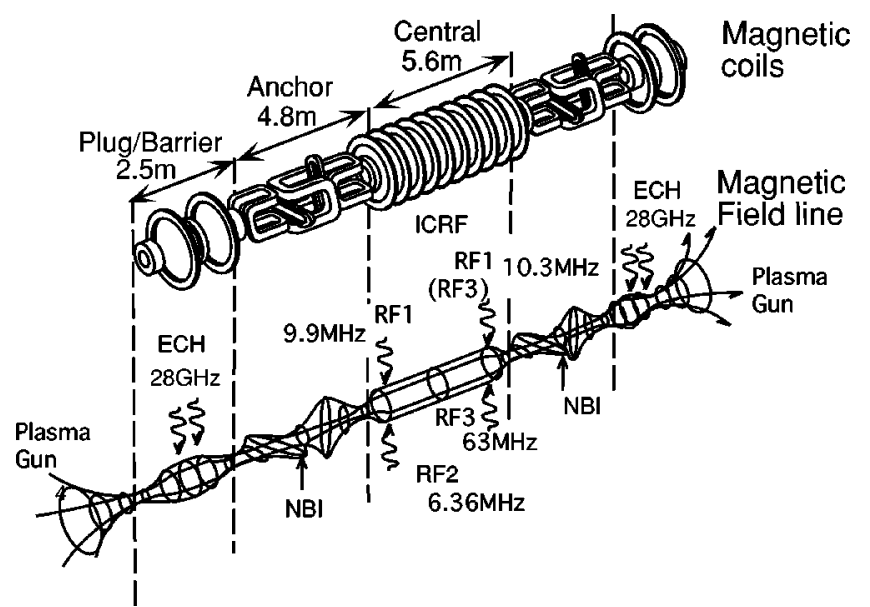

FIG. 1. Schematic drawing of the coil system and magnetic field lines. The location of the heating systems is indicated.

ends. Recently, conducting plates to fix the potential at the plasma boundary were installed near the surface of the plasma in the fanning magnetic flux tube of the transition regions on both sides of nonaxisymmetric minimum- $B$ anchor cells. With four discrete pairs of plates, the spacing is set so as to follow the shape of the magnetic flux tube that maps to a circular magnetic flux tube of 0.4 m diameter at the central cell midplane. ${ }^{4}$ The positive potential for the ion confinement is formed in the axisymmetric end mirror cells by injecting fundamental $\mathrm{ECH}$ with a frequency of $28 \mathrm{GHz}$. Before a modification of ECH antennas, the radiation pattern of the microwave beam from the antenna on the resonance layer was nonaxisymmetric because the microwave beam was directly injected with an angle of about $50^{\circ}$ respect to the machine axis. Between the conventional Vlasov-type antenna and the resonance layer, a cylindrical reflector has been inserted and adjusted its position and focal length. With the modification, the radiation pattern becomes axisymmetric and the potential profile also becomes axisymmetric. ${ }^{9}$

The strength of the magnetic field at the midplane of the central cell and the anchor cell is typically 0.4 and $0.6 \mathrm{~T}$, respectively. The stainless steel limiter with a radius of 0.18 $\mathrm{m}$ is located near the midplane. The short pulse $(1 \mathrm{~ms})$ gunproduced plasmas are injected initially from both ends and the main discharge is sustained with ICRF power in combination with the hydrogen gas puffing in the central cell. The maximum discharge duration of $0.5 \mathrm{~s}$ is limited by the ICRF power supply.

\section{B. ICRF system}

ICRF is commonly used for the plasma production in the tandem mirror experiments. ${ }^{10-12}$ In the GAMMA, three ICRF systems (RF1, RF2, and RF3) are used in the present experiments. An RF1 system with frequencies of 9.9 and 10.3 MHz is used for the ion heating in the anchor cell and the plasma production in the central cell. RF1 has two final outputs and supplies the power to antennas on both east and west sides of the central cell. The frequencies of RF1 (9.9, $10.3 \mathrm{MHz}$ ) are adjusted to the fundamental cyclotron resonance frequency near the midplane of the anchor cell. The frequencies of RF1 on both sides are slightly different from each other in order to avoid the strong interference. The RF2 system that has also two final outputs with the frequency of 6.36 MHz is used for the ion heating in the central cell. The frequency $(6.36 \mathrm{MHz})$ is adjusted to the fundamental cyclotron resonance frequency near the midplane of the central cell. The radiated power of RF1 and RF2 is typically around $100 \mathrm{~kW}$. The effect of RF1 for the central cell plasma heating is small because the fundamental resonance layer for RF1 exists near the end of the central cell where the magnetic field gradient is quite steep. The effect of RF2 for the plasma production is also small. The density is almost independent of the RF2 power. A new ICRF system (RF3) that has one output with higher frequency $(36-76 \mathrm{MHz})$ is fabricated to produce the high-density plasma.

So-called Nagoya-type III antennas for RF1 and conventional double half-turn antennas for RF2 are installed on both ends of the central cell. Two output of RF1 (RF2) are connected to both east and west sides of the antennas. Nagoyatype III antennas have four plate elements surrounding the plasma column. The rf current on each plate flows with a phase difference of $90^{\circ}$ and generates a rotating electromagnetic field in the direction of the electron cyclotron motion $(m=+1)$. These plates consist of two pairs: one with vertical plates and the other with horizontal plates. When the power of RF1 into the four plates is equally increased, the profile of the floating potential of the limiter that is segmented into eight sections shows a nonuniformity of the plasma in the azimuthal direction. ${ }^{13}$ The relation between the floatiing potential profile and the density profile measured by an azimuthal array of the electrostatic probes is discussed in Ref. 13. The reduction of the diamagnetic signal related to the nonuniformity of the floating potential is also observed. It is suggested that the effect of the nonaxisymmetry of the elongated fan region between the central cell and anchor cell is essential for this nonuniformity. In this experiment, only a vertical pair of the east antenna and a horizontal pair of the west antenna are used.

\section{PLASMA PRODUCTION WITH HIGH HARMONIC ICRF WAVES}

\section{A. Eigenmodes of fast Alfvén waves}

In GAMMA 10 experiments, RF1 is used for the plasma production in combination with the gas puffing in the central cell. Because RF1 is used also for the ion heating in the anchor cell, the frequency of RF1 is restricted near the cyclotron resonance frequency near the midplane of the anchor cell. In present experimental conditions of the GAMMA 10, that is, the relatively low density of $2 \times 10^{18} \mathrm{~m}^{-3}$, the plasma diameter of $0.36 \mathrm{~m}$ and the frequency just above the fundamental cyclotron frequency, one radial eigenmode of fast Alfvén wave with an azimuthal mode number of $m=+1$ is only excited in the central cell plasma. Eigenmodes excited in the central cell have been calculated by using a simple model, where the plasma is assumed to be cold, cylindrically uniform and surrounded by a conducting wall. Figure 2 shows the calculated dispersion relation under the fixed frequency of $9.9 \mathrm{MHz}$ in the central cell. It is clearly indicated 


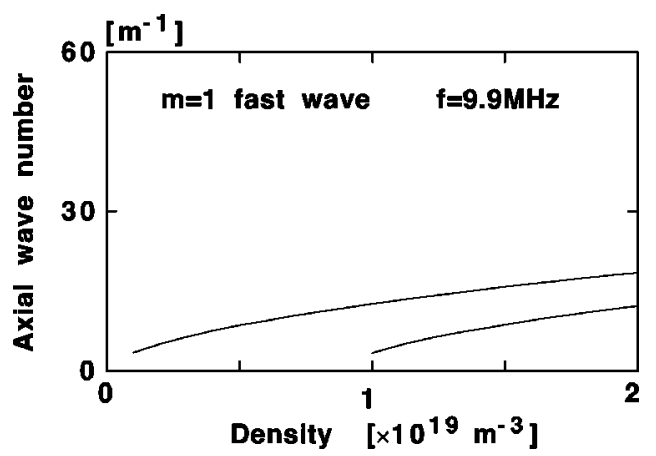

FIG. 2. The dispersion relation of $m=+1$ fast Alfvén waves under the fixed conditions of the frequency $9.9 \mathrm{MHz}$, magnetic field strength $0.4 \mathrm{~T}$, and plasma radius $0.18 \mathrm{~m}$.

that only one mode can be excited below the density of $10^{19} \mathrm{~m}^{-3}$. The second radial mode appears from the density above $10^{19} \mathrm{~m}^{-3}$. The axial wave number in the present experimental conditions is less than 5, as shown in Fig. 2. The central cell length corresponds to two to four times the wavelength of RF1. When there is nonuniformity of the magnetic field strength and plasma parameters in the axial direction, the wave excitation is affected from the axial boundary conditions and eigenmodes are also formed. The excitation of the axial eigenmodes depends strongly on the density. The existence of the axial eigenmodes means the wave field becomes strong discretely when the density changes. When the number of eigenmodes is small, it is possible the density is clamped to a certain value on which the axial eigenmodes is formed strongly.

High-density plasma production by the helicon waves with the frequency above 20 times cyclotron frequency near the midplane of the central cell was reported in the HIEI tandem mirror ${ }^{14}$ and the density of above $10^{19} \mathrm{~m}^{-3}$ has been realized in the experiments. The new RF3 system has the frequency range of 36-76 MHz, which corresponds to 6-12 times cyclotron frequency at the central cell midplane and the maximum output power and pulse length are $200 \mathrm{~kW}$ and $500 \mathrm{~ms}$, respectively. In the present experiment, the frequency of $63 \mathrm{MHz}$ is selected as the tenth harmonic resonance exists near the midplane. The calculated dispersion relation is shown in Fig. 3. Several radial eigenmodes are excited at the present density range and the number of the

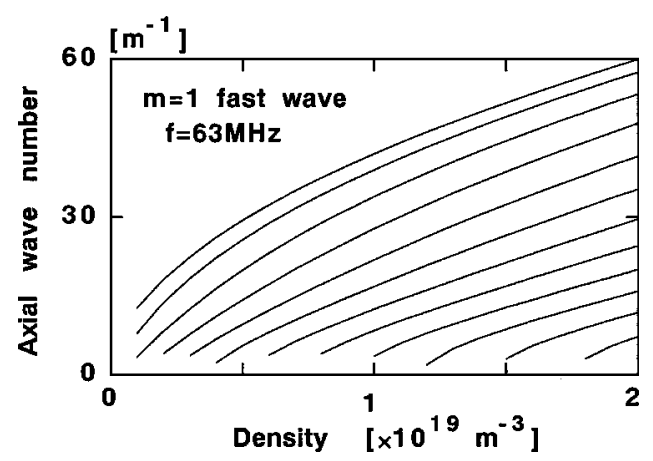

FIG. 3. The dispersion relation of $m=+1$ fast Alfvén waves under the same conditions as of Fig. 2, except for the frequency. The frequency is 63 $\mathrm{MHz}$.

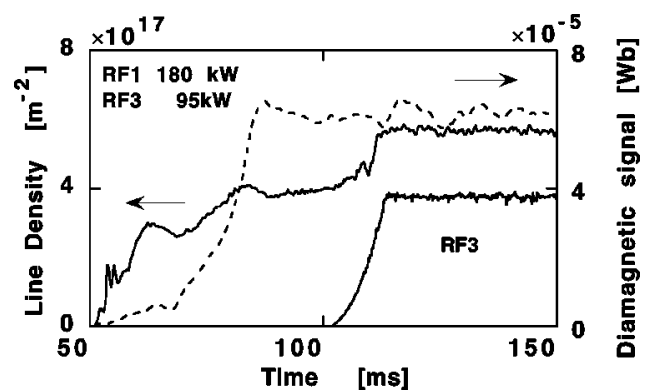

FIG. 4. Typical temporal evolution of the line density and diamagnetic signals when the RF3 power is injected, as indicated in the figure.

modes increases as the density increases. Those modes will also form eigenmodes in the axial direction. Because those eigenmodes excited strongly at various densities, the density can increase more smoothly than in the case of $9.9 \mathrm{MHz}$. It is expected the density clamping due to the eigenmodes excitation is avoidable by using a higher-frequency ICRF source.

\section{B. Experimental results with high harmonic ICRF waves}

The preliminary experiments with the RF3 have been performed. The frequency of $63 \mathrm{MHz}$ is selected as the tenth cyclotron higher harmonic resonance exists near the midplane of the central cell. Both Nagoya-type III and double half-turn antennas are tested for launching the higherfrequency waves. There are small differences between them in the preliminary experiments. We need to have more experiments for the precise comparison. Figure 4 shows the typical time evolution of the line density and diamagnetism. The RF1 power is fixed at $180 \mathrm{~kW}$. In the case, the ion temperature of several $\mathrm{keV}$ are sustained with the RF2 and in the hot ion mode operation. The RF3 source is superposed from $100 \mathrm{~ms}$, as indicated in Fig. 4. An increase of the line density is clearly observed. When the RF1 power was increased under the same conditions as in Fig. 4, that is, the amount of gas puffing, the RF2 power, and so on, we observed little increase on the line density. In the previous experiments ${ }^{15}$ on which a large amount of gas puffing was used in the low magnetic field operation, we have observed the density increase up to $1 \times 10^{19} \mathrm{~m}^{-3}$ accompanied with a large reduction of the diamagnetism. The ion temperature reduced to less than $100 \mathrm{eV}$. In the present RF3 case as shown in Fig. 4, no reduction of the diamagnetism is observed with an increase of the density. Those results indicate the density clamping due to the eigenmode excitation of RF1 was possible in the GAMMA 10 central cell. When the confinement potential is formed, the density is expected to increase smoothly with RF3.

The increasing rate of the line density depends on the RF3 power as shown in Fig. 5. The increasing rate of the line density becomes larger with an increase of the power. If the gap between densities for the eigenmodes formation of RF1 is filled up by the density increase with RF3, the eigenmodes excitation of RF1 is expected in the higher-density region. The density profile measurement indicates that the increase of the density occurs mainly in the core region of the plasma. 


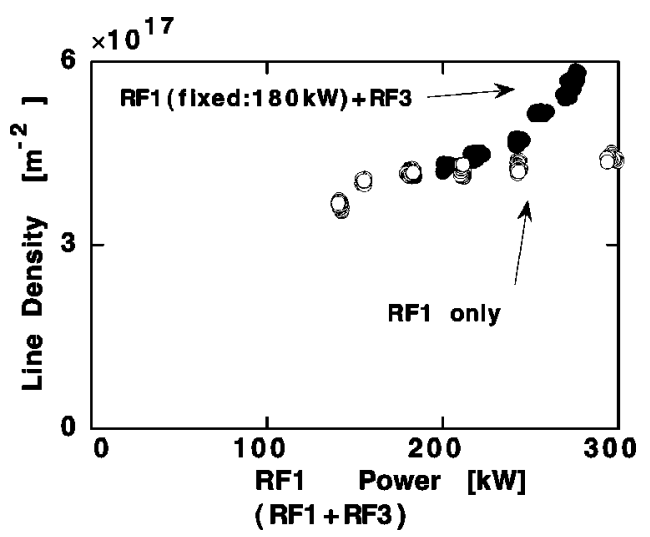

FIG. 5. RF1 power dependence of the line density (open circles) and $(\mathrm{RF} 1+\mathrm{RF} 3)$ power dependence of the line density with fixed RF1 power of $180 \mathrm{~kW}$ (closed circles).

As previously mentioned, there exists a higher harmonic resonance layer near the midplane. The frequency corresponds to the tenth ion cyclotron harmonic frequency near the midplane of the central cell. The high-energy ions of which energy is above $50 \mathrm{keV}$ are detected in the end loss with RF2 heating ${ }^{16}$ before RF3 pulse. It is expected that the high-energy ions are produced due to higher harmonic resonance. Figure 6 shows the signal of ccHED (central cell high-energy ion detector) installed at the midplane. ${ }^{17}$ The ccHED is set just outside the limiter radius and can detect the ions of which energy is above $10 \mathrm{keV}$. When the RF3 pulse is applied, the signal of high-energy ions increases with the RF3 power. Because there are no increases in the diamagnetic signal, a very small part of high-energy ions will be accelerated by RF3.

\section{HIGH-DENSITY PLASMA PRODUCTION WITH POTENTIAL CONFINEMENT}

The density increase due to the potential formation is clearly observed in the GAMMA 10 tandem mirror. ${ }^{4}$ The typical wave forms of the plugging experiment are shown in Fig. 7. When ECH only on the east or west plug/barrier cell is applied, the reduction of the end loss current on the ECH side and the increase of the end loss current on the opposite

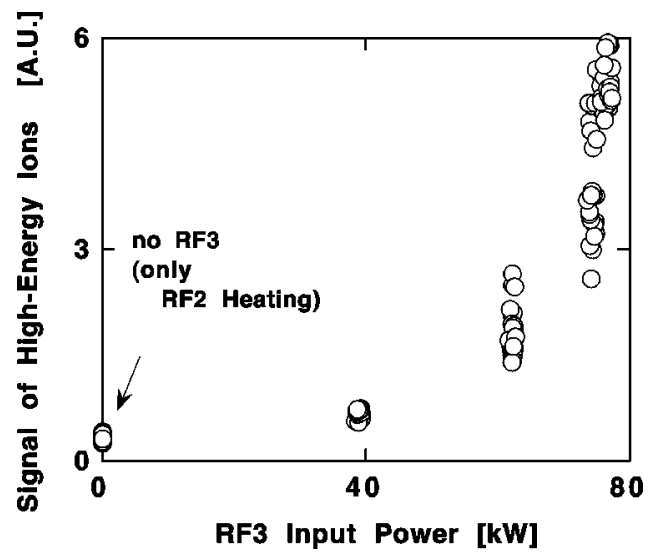

FIG. 6. RF3 power dependence of the signals of the high-energy ions (ccHED).

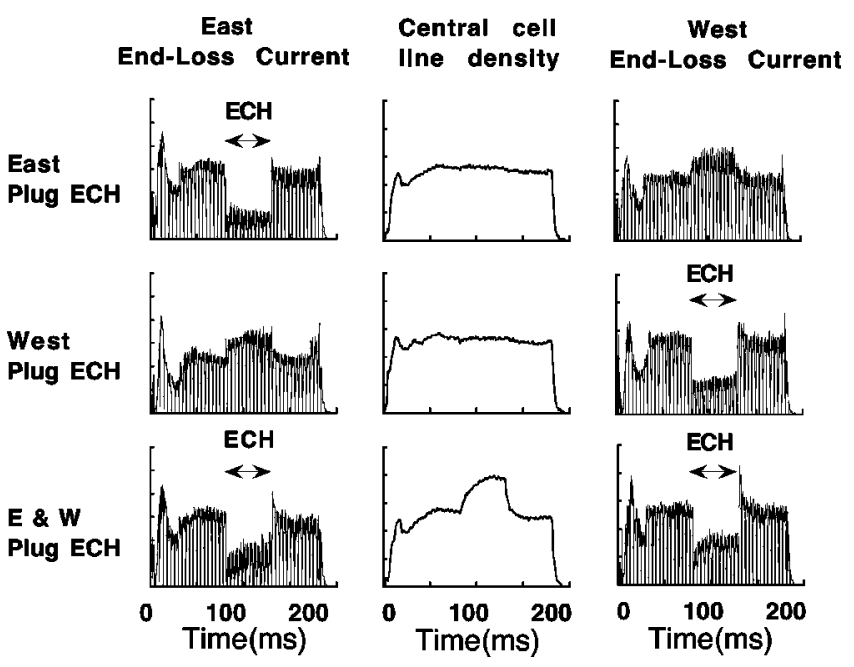

FIG. 7. Typical wave forms of the plugging experiment when ECH is applied (a) only on the east plug/barrier cell, (b) only on the west plug/barrier cell, and (c) both on the east and west plug/barrier cells.

side are illustrated in the figure. There are no increases of the central cell line density. When both east and west ECH are applied, the clear increase of the central line density is indicated. The density increase due to potential confinement depends on the initial density and the limit of the increase is observed in the previous experiment. ${ }^{5}$ It was difficult to increase the central cell density higher than $2.7 \times 10^{18} \mathrm{~m}^{-3}$ with and/or without potential confinement. When the initial density is low, the increase of more than $100 \%$ was attained. Figure 8 shows the density on an axis before ECH and during ECH as a function of the density before ECH. Closed circles indicate the central cell densities before ECH and closed triangles indicate those during ECH. Recently, NBI in the anchor cell became effective by reducing gas penetration from the injector to the plasma region with newly installed baffle plates between them. The plasma density in the anchor cell increased $70 \%$ with $25 \mathrm{kV}-20$ A NBI. However, the

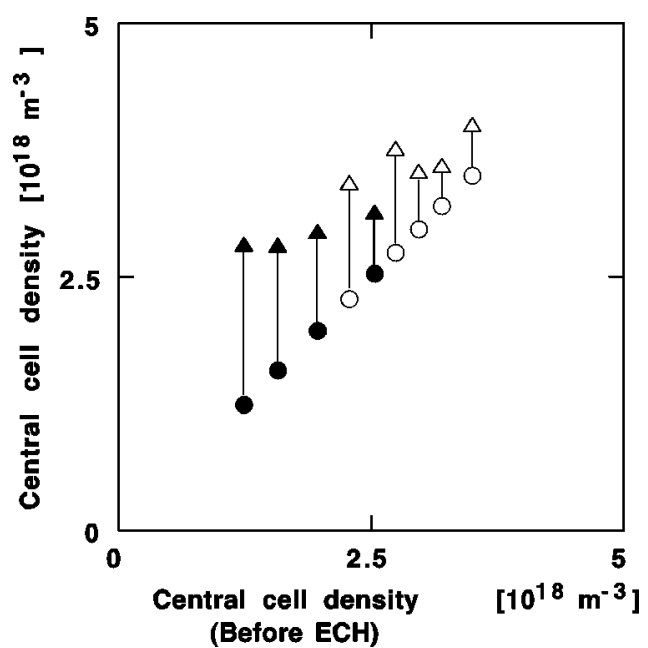

FIG. 8. Central cell density before ECH (circles) and during ECH (triangles) as a function of the density before ECH. Closed symbols indicate the previous experiments and open symbols indicate the experiments with RF3 and NBI. 
density started to decrease during NBI. The shine through of the beam is more than $90 \%$ under the condition of the line density of $5 \times 10^{17} \mathrm{~m}^{-2}$ in the anchor cell and causes a large amount of recycling gas. Experiments with the potential confinement on the higher-density plasma were just started with RF3 and NBI. In Fig. 8, open circles indicate the densities before $\mathrm{ECH}$ and open triangles indicate those during $\mathrm{ECH}$ in the experiment with RF3 and NBI. The initial density increased and the maximum density of $4 \times 10^{18} \mathrm{~m}^{-3}$ was attained, as indicated in Fig. 8. Though the density increment decreases with the initial plasma density, we expect a large increment at a higher-density region with the progress of wall conditioning.

\section{SUMMARY}

The improvement of potential confinement was attained in the GAMMA 10 tandem mirror by axisymmetrization of heating systems for the plasma formation, heating, and potential formation. However, the saturation of the attained density is observed. One of the possible mechanisms is the density clamping due to the eigenmode formation of ICRF waves in the axial direction. In the previous experiment, a radial eigenmode with an azimuthal mode number of $m$ $=+1$ is only excited in the central cell plasma. With a fast Alfvén wave (RF3) of which frequency is about tenth higher harmonic frequency at the midplane of the central cell, we observed a clear increase of the density. On the preliminary experiments with RF3 and NBI, the maximum density of 4 $\times 10^{18} \mathrm{~m}^{-3}$ was attained with the potential confinement. We expect a large increment at a higher-density region with the progress of wall conditioning.

\section{ACKNOWLEDGMENT}

The authors acknowledge the GAMMA 10 group at the University of Tsukuba for their collaboration.
${ }^{1}$ S. Miyoshi, T. Cho, H. Hojo et al., Proceedings of the 13th International Conference on Plasma Physics and Controlled Nuclear Fusion Research, Washington, 1990 (International Atomic Energy Agency, Vienna, 1991), Vol. 2, p. 539

${ }^{2}$ T. Tamano, T. Cho, H. Hirata et al., Proceedings of the 15th International Conference on Plasma Physics and Controlled Nuclear Fusion Research, Seville, 1994 (International Atomic Energy Agency, Vienna, 1995), Vol. 2, p. 399.

${ }^{3}$ Y. Kiwamoto, Y. Tatematsu, T. Saito et al., Phys. Plasmas 3, 578 (1996).

${ }^{4}$ K. Yatsu, L. G. Bruskin, T. Cho et al., Nucl. Fusion 39, 1707 (1999).

${ }^{5}$ K. Yatsu, T. Cho, M. Hirata et al., Proceedings of the 26th European Conference on Controlled Fusion and Plasma Physics, Marstricht, 1999 (European Physical Society, Petit Lancy, 1999), ECA 23J, p. 461.

${ }^{6}$ R. W. Boswell, Plasma Phys. Controlled Fusion 26, 1147 (1984).

${ }^{7}$ F. F. Chen, Plasma Phys. Controlled Fusion 33, 339 (1991).

${ }^{8} \mathrm{~K}$. Yatsu, T. Cho, M. Hirata et al., 18th IAEA Fusion Energy Conference, Sorrento, 2000, IAEA-CN-77/EXP1/10 (International Atomic Energy Agency, Vienna, 2000).

${ }^{9}$ T. Saito, Y. Tatematsu, Y. Kiwamoto et al., Fusion Eng. Design 53, 267 (2001).

${ }^{10}$ R. Breun, S. N. Golovato, L. Yujiri et al., Phys. Rev. Lett. 47, 1833 (1981).

${ }^{11}$ S. N. Golovato, K. Braw, J. Casey et al., Phys. Fluids 31, 3744 (1988).

${ }^{12}$ M. Ichimura, M. Inutake, S. Adachi et al., Nucl. Fusion 28, 799 (1988).

${ }^{13}$ M. Ichimura, S. Kanazawa, E. Ishikawa et al., Proceedings of the 1998 International Congress on Plasma Physics combined with the 25th European Physical Society Conference on Controlled Fusion and Plasma Physics, Praha, 1998, edited by P. Pavlo (European Physical Society, Petit Lancy, 1998), ECA 22C, p. 1292.

${ }^{14}$ H. Takeno, Y. Yasaka, O. Sakai, and R. Itatani, Nucl. Fusion 35, 75 (1995).

${ }^{15}$ M. Ichimura, S. Adachi, T. Cho et al., Proceedings of the 14th European Conference on Controlled Fusion and Plasma Physics, Madrid, 1987, edited by F. Engelmann and J. L. Alvarez Rivas (European Physical Society, Petit-Lancy, 1987), ECA 11D, Part II, p. 554.

${ }^{16}$ T. Saito, M. Sakakibara, Y. Kiwamoto et al., Rev. Sci. Instrum. 68, 1433 (1997).

${ }^{17}$ M. Ichimura, C. Satake, K. Sakata et al., Rev. Sci. Instrum. 70, 834 (1999). 